eine beschränkte klinische Prüfung keine Klarheit über den therapeutischen Wert eines Produktes schaffen kann.

2. Die Zentralstelle darf ein ungünstiges Urteil über ein Präparat, sei es nun ein Schwindelmittel, eine Spezialität oder ein Arzneimittel, weder veröffentlichen, noch der vorgesetzten Behörde zur weiteren Veranlassung (Strafverfolgung, Geheimmittelliste) mitteilen, ehe der Untersuchungsbefund dem betreffenden Fabrikanten oder Erfinder mitgeteilt ist, und dieser Gelegenheit zu einer Rückäußerung und ev. Richtigstellung, unter Umständen auch zu einer Änderung oder Zurückziehung des Präparates hatte.

3. Die Zentralstelle darf unter keinen Umständen die Untersuchungsbefunde über zur Prüfung gelangte Mittel, die zu einer Beanstandung keinen Anlaß geben, publizieren, es sei denn lediglich in Form der Aufstellung von Prüfungsvorschriften oder Identitätsreaktionen. Denn es besteht die Gefahr, daß ebenso wie dies mit Bezeichnungen: „Patentamtlich geschützt“, „Behördlich beglaubigt", „Von Arrten und Professoren begutachtet" usw. geschieht, eine einfache Bestätigung der Zentralstelle zu Reklamezwecken ausgenutzt werde und so im direkten Gegensatze zu ihrem eigenen Zwecke zur Täuschung des Publikums und vielleicht auch des Arztes über den Heilwert der betreffenden Präparate diene.

4. Weiterhin muß meines Erachtens noch verlangt werden, daß mit der Schaffung einer Zentralstelle diejenige einer übergeordneten Behörde Hand in Hand gehe, die nicht nur als Beschwerdeinstanz djenen könnte, in den Fällen (und diese werden recht häufig sein), in welchen die Richtigkeit des Befundes der Zentralstelle durch den Fabrikanten bestritten wird, sondern in deren Hand auch dio Entscheidung darïber zu liegen hätte, welche Folgen dem betreffenden Befund des Untersuchungsamtes zu geben seien.

Naturgemäß liegt in einem abfälligen Urteil einer derartigen offiziellen Prüfungsstelle eine außerordentlich große materielle Schädigung für den betreffenden Fabrikanten, unter Umständen eine Diskreditierung seiner Firma, vielleicht eine Vernichtung seiner Existenz. Die Folgen einer Bokanntmachung eines solchen Untersuchungsbefundes sind deshalb unter Umständen von viel größerer Tragweite, als wie sie der Leiter des betreffenden Instituts, der ja meist mit relativ jungen Assistenten arbeiten muB, in Erkenntnis des Wortes ,errare humanum est" wird verantworten können, umsomehr, als auch die besten analytischen Methoden versagen können, wie dies beispielsweise bei der offizinellen Theobrominbestimmung der Fall ist. Diese Verantwortung muB ihm eine übergeordnete Instanz abzunehmen imstande sein, deren Bekanntmachungen andererseits auch eine viel größere Wirkung besitzen würden. Haben doch auch in der Puroaffaire die analytischen Befunde von $G$ e $r$ e $t$, Hutchins on usw. erst Nachdruck erhalten und Konsequenzen mit sich gobracht, nachdem sie durch eine erste Autorität wie Geheimrat G r u be r mittels absolut eindeutiger neuer biologischer $\mathrm{Me}$ thoden bestätigt worden sind.

Diese zweite Instanz hätte dann gleichzeitig zu entscheiden, ob ein Präparat, sei es wegen Gesund- heitsschädlichkeit, sei es wegen betrügerischen Charakters, völlig zu verbieten, oder wegen schwindelhafter Angaben auf die Geheimmittelliste zu setzen sei, ob eine Warnung an den Fabrikanten, eine öffentliche Festnagelung oder gar eine Mitteilung an den Stratsanwalt angezeigt sei, oder ob und in welcher Form eine offizielle Publikation des Untersuchungsbefundes stattfinden solle. Eine solche Instanz, die dann tatsächlich, gestützt auf die Ergebnisse der Zentralstelle die Beraterin des Bundeates bei der Aufstellung der Geheimmittelliste wäre, würde zweckmäßig aus einer Kommission von Sachverständigen gebildet, wie sie bereits der Gesetzentwurf (allerdings mit beschränkteren Kompetenzen und nur beratender Stimme) vorsieht. Nur von einer derartigen, alle Garantien sachgemäßer Erledigung bietenden Einrichtung, an der sie selbst, durch Entsendung einiger ihrer Mitglieder in diese Kommission aktiv beteiligt sein müßte, nicht aber von einer einseitigen Zensurbehörde kann die deutsche Industrie ohne Schädigung ihre eigenen Interessen das erwarten, was sie selbst am allermeisten erstrebt, die $\mathrm{R}$ e i nigungdes deutschen Arzneimittelmarktes von unlauteren, widerrechtlich unter der Flagge "synthetischer Arzneimittel ${ }^{\text {sc }}$ segelnden Präparaten, die A usmerzung betrügerischer oder skrupelloser Fabrikanten aus ihren eigenen Reihen.

\section{Über Pilzgifte in Getreide, Wuirzen und Hefen und die Abhängigkeit ihrer Wirkung von Mineralsalzen *).}

\author{
Von Dr. F. Hayduch-Berlin. \\ (Eingeg. d. 26.j6. 1908.)
}

M. H.! Die Rohstoffe der Gärungsgewerbe und die daraus bereiteten Maischen und Würzen enthalten bekanntermaßen Stoffe, die wir als Pilzgifto bezeichnen können, da sie schon in verhältnismäßig geringer Konzentration das Leben und die Tätigkeit der in der Gärungstechnik verwendeten Pilze beeinträchtigen. So finden wir in Gerste und Malz organische Säuren verschiedener Art, weiter Gerb- und Bitterstoffe, die vorwiegend auf die Spelzenanteile des Korns lokalisiert sind, ja sogar ein Alkaloid, das Hordenin der Gerste, das zwar als ungiftig gilt, aber seiner Zusammensetzung nach zu einer Verbindungsgruppe gehört, deren vielseitige Giftwirkungen bekannt sind. Im Hopfen haben wir an Stoffen der charakterisierten Art wiederum Gerbstoffe, weiter Harze und Säuren verschiedener Natur. In der Würze können außer den von den Rohstoffen übernommenen Stoffen enthalten sein Metalle, wie z. B. Eisen aus dem Brauwasser oder Kupfer aus den Hefezucht- und Sudhausapparaten. Zu der Maische in der Brauerei setzen wir sogar künstlich Stoffe oder erzeugen sie durch absichtlich eingeleitete Bakterientätigkeit,

*) Vortrag, gehalten in der Sitzung der Fachgruppe für Gärungschemie gelegentlich der Hauptversammlung des Vereins deutscher Chemiker in Jena 1908. 
die scharfe Pilzgifte darstellen, wie Milchsäure, Schwefelsäure, Ameisensäure, Flußsäure, um der Hefe einen Schutz gegen schädliche Mikroorganismen zu gewähren. Aber auch die Hefe ist nicht unempfindlich gegen diese Antiseptika, sondern erliegt ihrer Wirkung, wenn deren Konzentration über ein gewisses Maß hinaus gesteigert wird. Endlich entstehen durch die Enzymtätigkeit der Hefe währond der Gärung Alkohol, Kohlensäure, Glycerin, Bernsteinsäure, Oxalsäure, beim Vorhandensein anderer Mikroorganismen die für sie typischen Säuren wie z. B. Milchsäure, Buttersäure, Essigsäure. Wir sehen also, daß die Hefe in Würzen und Maischen reichliche Gelegenheit hat zur Aufnahme von Stoffen die ihrem Dasein gefährlich werden können, und daß alle die wunderbaren Gärungserscheinungen, die wir im Gärungsgewerbe beobachten, wie z. B. die stillstehende Gärung in der Brauerei, schlechte Vergärung und Schaumgärung in der Brennerei, geringe Hefenausbeute in der Preßhefefabrik, in der Wirkung solcher Pilzgifte wohl ihre Erklärung finden könnten.

Es soll aber heute nicht meine Aufgabe sein, den Pilzgiften der genannten Art im einzelnen nachzugehen und die Möglichkeit ihrer Wirkung auf die Hefen in der Praxis nachzuprüfen, sondern ich will diese Reihe erweitern durch die Beschreibung einer Giftwirkung auf Hefe, die wir bei Arbeiten im Institut für Gärungsgewerbe in Berlin beobachtet haben und deren Zurückführung auf gewisse Eiweißstoffe uns in einer Reihe von Untersuchungen gelungen ist. Die Stoffe, von denen hier die Rede sein soll, besitzen eine so weite Verbreitung, daß wir geneigt sind, auf ihre überaus scharfe Giftwirkung gegen Hefe einen großen Teil der abnormen Gärungserscheinungen, die wir in der Praxis beobachten, zurückzuführen.

Die erste derartige Beobachtung wurde vor etwa drei Jahren gemacht, gelegentlich einer von $\mathrm{D}$ e $1 \mathrm{~b} \mathrm{r} \mathrm{ü} \mathrm{c} \mathrm{k} \mathrm{geleiteten} \mathrm{und} \mathrm{von} H$. L a $\mathrm{n}$ g e u. a. ausgeführten Arbeit, bei der es sich darum handelte, den physiologischen Zustand der Hefe, d. h. ihren Enzymgehalt und ihre Fähigkeit, Enzyme hervorzubringen, zu beeinflussen durch Veränderung der Temperatur-, Ernährungs- und Lüftungsbedingungen, sowie durch Darbietung von Reizstoffen, worunter Stoffe zu verstehen sind, welche die Lebenstätigkeit der Hefe erhöhen, ohne daß diese Wirkung auf Ernährungsvorgänge zurückzuführen wäre. Der Einfluß von Reizstoffen auf die Zymasetätigkeit der Hefe wurde mittels der Triebkraftbestimmungsmethode nach $\mathrm{M}$. H a y d u ck studiert in der Weise, daß die zu prüfenden Stoffe zusammen mit der Hefe der aus destilliertem Wasser hergestellten Rohrzuckerlösung zugesetzt und dann im Verlauf von zwei Stunden die von der Hefe entwickelten Kohlensäuremengen gemessen wurden. Unter den Reizstoffen nahmen verschiedene Getreidearten eine hervorragende Stelle ein, indem sie, in Form von Schrot oder als wässeriger Auszug daraus der Rohrzuckerlösung hinzugefügt, die Triebkraft von Brennerei- und Preßhefen ganz außerordentlich steigerten. Dieselben Versuche wurden nun auch mit untergäriger Bierhefe angestellt, allein hierbei trat etwas ganz Merkwürdiges ein : Die Triebkraft der Hefe wurde durch Zusatz von Roggenschrot nicht erhöht, sondern im Gegenteil auf ein Mini- mum herabgedrückt. Diese Erscheinung war, wie He n n e b r g bald darauf nachwies, verbunden mit einem rapiden Absterben der Hefezellen, ja es wurde beobachtet, daß unter Umständen in 5-7 Minuten bis zu 99\% der Zellen abgetötet wurden.

M. H.! Diese Giftwirkung war höchst rätselhaft: ein so harmloser, ja man sollte meinen allseitig bekömmlicher Stoff, wie das reife Roggenkorn, enthält scharfe Hefengifte! Wie verhalten sich denn unsere anderen Getreidearten in dieser Beziehung? war naturgemäß die erste Frage, die sich aus unserer Beobachtung ergab. Es wurden nun der Reihe nach untersucht Weizen, Gerste, Mais und Hafer, wobei sich ergab, daß die ersten beiden Getreidearten ebenfalls in hohem Maße die Giftwirkung besaßen, die letzten beiden dagegen nicht, sondern vielmehr die Triebkraft der Bierhefe steigerten.

Die weitere Beobachtung, daß auch Pepton Witte und Hühnereiweiß, zwei hoch eiweißhaltige Stoffe, die Bierhefe unter den beschriebenen Versuchsbedingungen vergifteten, führte uns auf die Vermutung, daß der wirksame Bestandteil unter den Eiweißstoffen zu suchen ist, vielleicht unter den Nucleinen, deren baktericide Eigenschaften ja bekannt sind. Im Verfolg dieser Idee wurde nun zunächst versucht, durch mechanische Behandlung des Getreides giftige Eiweißstoffe zu isolieren. Der Erfolg war ein günstiger. Aus einer wässerigen Aufschwemmung von Roggenschrot in Wasser ließ sich nach Absetzen der schweren, zumeist aus Stärke und Spelzen zusammengesetzten Bestandteile, ein fein in der Flüssigkeit schwebender Anteil des Roggens abfiltrieren, der seiner schlammartigen Konsistenz und seiner starken Eiweißreaktion nach die Hauptmenge des Roggeneiweiß enthalten mußte. Dieser Eiweißschlamm war von hervorragender Giftigkeit gegen die Bierhefe. An Wirkung gleich erwies sich der aus Weizenmehl durch Auskneten mit Wasser gewinnbare Kleber. Eine weitere Stütze unserer Vermutung wurde darin gefunden, daß von der ganzen Roggenpflanze nur die Eiweißspeicher, die Körner, den Giftstoff enthielten, nicht dagegen die Wurzeln, Halme, Grannen und Spelzen.

Trotz dieser Ergebnisse erschien es notwendig, das Getreide auch auf die im Anfang meines Vortrags genannten Stoffe zu prüfen, die zwar keinen integrierenden Bestandteil des Korns bilden, ihm aber vielfaeh von der Natur als Antiseptika mitgegeben werden, wie z. B. organische Säuren, Alkaloide, ätherische Öle, Glucoside usw. Allein es wurde durch Behandlung des Getreides mit Alkohol, Ather, Wasserdampf, kurzum durch Arbeitsmethoden, mittels welcher diese Stoffe isoliert werden, keine Spur einer für Hefe giftigen Substanz gewonnen, so daß auch dieses negative Ergebnis uns in unserer alten Auffassung bestärkte.

Den mechanischen Trennungsversuchen folgten chemische, zunächst ohne jeden Frfolg. Das Weizenmehl, das, direkt mit der Hefe zusammengebracht, eine so fabelhaft intensive Wirkung ausübte, gab an destilliertes Wasser, selbst bei längerer Behandlung damit, kaum nennenswerte Mengen eines Giftstoffs ab. Besser glückte schon die Extraktion des Weizenmehls mit Glycorin, mit dem sich ein ziemlich giftiger Auszug herstellen ließ. Die Überlegung, daß doch aber der Giftstoff, um auf 
die Hefe in Rohrzuckerwasser wirken zu können, in wasserlöslicher, diffusibler Form vorhanden sein, daß also, da Wasser allein die Lösung nicht bewirkt, die Hefe selbst sich daran beteiligen muB, führte mich zu einer Arbeitsmethode, die von gutem Erfolg begleitet war. Es gelang nämlich, durch Behandeln einer wässerigen Weizenmehlaufschwemmung mit mittels Sand zerriebener Bierhefe, im Verlauf einer kurzen Zeit einen stark giftigen Auszug zu erhalten. Wenn der Giftstoff unter den Eiweißstoffen des Korns zu suchen war und - das war der nüchstliegende SchluB — durch die Peptase der Hefe abgebaut und in die wässerige Lösung übergeführt wird, so mußte man dasselbe mit Hilfe eines eiweißverdauenden Enzympräparats erzielen können. Das war in der Tat der Fall : auch durch Verwendung von Pepsin an Stelle von zerriebener Hefe wurde ein stark giftiger Auszug aus Weizenmehl erhalten, und zwar in noch kürzerer Zeit als in dem ersten Falle.

Die beste Methode aber zur Extraktion des Giftstoffs aus dem Weizenmehl fand ich in der Behandlung des Mehls mit verd. Salzsäure $(0,1 \%)$. Ein solcher Auszug ließ sich im Verlauf einer halben Stunde herstellen und besaß nach der Neutralisation mit Natronlauge eine Giftwirkung gegen Bierhefe, welche an Intensität noch diejenige des unmittelbar mit der Hefe in Zuckerlösung zusammengebrachten Weizenmehls übertraf. Mit solchen Auszügen gelang es, die Bierhefe innerha,lb von wenigen Minuten bei Gegenwart von Zucker quantitativ zu töten.

Die Versuche, den wirksamen Eiweißstoff aus den Weizenauszügen durch Fällung in reinerer Form zu gewinnen, schlugen bis auf einen fehl, der in folgender Weise ausgeführt wurde. Bei der Neutralisation von Säureauszügen aus Weizenmehl mit Natronlauge fiel ein sehr fein verteilter Eiweißniederschlag aus, der bei $85^{\circ}$ zusammenflockte und sich dann leicht abfiltrieren ließ. Dieser Niederschlag, allerdings auch der davon abfiltrierte Auszug, wirkte stark giftig auf die untergärige Bierhefe ein.

Bis hierher sind wir auf dem Wege zur Identifizierung des neuen Hefepilzes gekommen, und ioh balte es für unwahrsoheinlich, daß es uns Gärungschemikern gelingen wird, eine genaue Feststellung dieses Stoffs zu erreichen, daß hierzu vielmehr die spezielle Erfahrung eines Physiologen auf dem Gebiet der Eweißstoffe gehören wird. Unsere jetzige Auffassung geht, noch einmal in kurzen Worten zusammengefaßt, dahin, daß das neue Hefengift zu den Eiweißstoffen oder deren Abbauprodukten zählt.

Gleich im Anfange unserer Arbeit drängte sich uns naturgemä $B$ die Überzeugung auf, da $\beta$ die $B e-$ obachtung des neuen Hefengifts in den Körnerfrüchten von größter Tragweite für die mit diesen Rohstoffen arbeitenden Gärungsgewerbe sein müsse. Wir setzten daher unverzüglich mit den Vorarbeiten ein, welche für die nachfolgende Durchforschung der Brauerei, Brennerei und Preßhefefabrik nach aus dem Getreide stammenden Hefengiften die notwendige Grundlage bildeten. Vor allem kam es darauf an, die Bedingungen zu studieren, unter denen eine Giftwirkung eintritt oder ausbleibt, unter denen der Giftstoff beständig ist oder zerstört wird.
Zunächst der Einfluß der Temperatur. Weizenund Roggenschrot wurden auf $105^{\circ}$ erhitzt; dabei ergab sich, daß das erstere seine alte Giftwirkung auf Hefe beibehält, während diejenige des letzteren vollkommen verschwindet. Wir sehen daraus, daß die giftigen Bestandteile der Körnerfrüchte nicht alle die gleiche Konstitution haben können, daß wir daher nicht von $\mathrm{d}$ e m Giftstoff, sondern nur von Giftstoffen sprechen dürfen, die allerdings in den meisten Beziehungen sich gleich verhalton. Das Weizenmehl vertrug weiter sogar ein längeres Kochen mit Wasser, ohne dabei seine Giftwirkung einzubüßen.

Nun wurden die verschiedenen Getreidesorten vermälzt in der Voraussicht, daB die Giftstoffe als Eiweißkörper durch die natürlichen tryptischen Kräfte des keimenden Kornes abgebaut und unwirksam gemacht werden. Allein das Mälzen verlief nur teilweise in diesem Sinne: Nur eine geringe $\mathbf{A b}$ nahme der Giftwirkung des Getreides konnte beobachtet werden, ja beim Hafer, der bis dahin als ungiftig galt, wurden durch das Mälzen die Giftstoffe erst erzeugt oder freigemacht. Beim Darren büßte das Gerstenmalz wieder einen Teil seines Giftstoffs ein, aber auch jetzt noch genügte der Rest, um das Leben der Hefe auf das schwerste zu schädigen. Naturgemä $\beta$ war der Gehalt an wirk samen Bestandteilen im Darrmalz abhängig von der Darrtemperatur, der es ausgesetzt gewesen, so daB also nur helles Malz die. Giftstoffe enthielt, während die Wirkung abnahm, je höher die Darrtemperatur, je dunkler das Malz war. Bei ausgesprochen dunklem Malz wurde keinerlei Giftwirkung gegen Bierhefe beobachtet.

Beim Maischen trat eine ganz merkwürdige Erscheinung zutage: Maischen aus Gerstendarrmalz oder Weizengrünmalz und destilliertem Wasser wirkten, selbst wenn sie gekocht wurden, scharf giftig auf Bierhefe; wurden aber nur die blanken Maischefiltrate verwendet, so war die Giftwirkung spurlos verschwunden, d. h. doch nicht spurlos, denn sie fand sich vor in den abfiltrierten Maischetrebern. Soll man danach nun sagen, der Giftstoff sei gar nicht in Lösung gegangen? Ohne Zweifel war er es, aber die Lösung war durch das Eiweißenzym zu weit getrieben, der Giftstoff war abgebaut und zerstört worden, wie ich sogleich zeigen werde.

Nach dem zuletxt Mitgeteilten erscheinen die Giftstoffe sehr widerstandsfähig, mitunter so sehr, daB man geneigt ist, ihre Eiweißnatur anzuzweifeln. Allein die $\mathbf{Z}$ weifel verschwinden wieder, wenn man folgendes Verhalten derselben in Betracht zieht. Ein giftiger Weizenauszug läßt sich durch kurzes Kochen mit ganz geringen Mengen von Säuren oder Alkalien $(0,1 \%)$ vollkommen seiner Wirkung berauben. Eine Peptonlösung wird durch Trypsin (Merck) verdaut; im Verlauf dieses Vorganges verschwindet die Giftwirkung der Lösung gegen Bierhefe. Aus diesem Versuch entnehme ich die Berechtigung zu der Erklärung für die Ungiftigkeit der vorhergenannten Maischefiltrate : Der Giftstoff bleibt nicht vollständig in den Trebern zurück, sondern es wird ein Teil gelöst, aber so weit abgebaut durch die tryptischen Kräfte des Malzes, daß er keine Giftwirkung mehr aufweist.

Wir kommen jetzt zu einer anderen Möglichkeit, die Wirkung des Hefengifts a bzuschwächen oder ganz 
aufzuheben. Versuchen Sie einmal, eine Bierhefe mit Weizenmehl in einer Rohrzuckerlösung zu vergiften, die nicht aus destilliertem Wasser, sondern aus einem kalkreichen Leitungswasser hergestellt ist; Sie werden eine große Enttäuschung erleben, denn die Hefe ist unter diesen Umständen vollkommen unempfindlich gegen das Gift. Im Anschluß an diese Beobachtung haben wir eine ganze Reihe von Salzen auf ihre giftvernichtende Kraft geprüft und dabei gefunden, daß kohlensaurer Kalk und Soda die Giftwirkung vollkommen, Gips und Chlorcalcium sie nahezu aufheben. Dann folgen Barium- und Zinksalze, was um so merkwürdiger ist, als diese Salze als Hefengifte bekannt sind, so daß sich also hier zwei Hefengifte in ihrer Wirkung paralysieren. Noch schwächer hob auf Magnesiumsulfat und endlich ohne erhebliche Unterschiede die Sulfate, Chloride und Phosphate der Alkalien. Die Entgiftung wird offenbar nicht von allen Salzen in derselben Weise ausgeführt. Während z. B. von den Kalksalzen schon ein Gehalt von wenigen Hundertstel Prozenten in der Zuckerlösung jede Giftwirkung unmöglich machen, kann dasselbe mit den Sulfaten, Chloriden und Phosphaten der Alkalien nur bei einem Zusatz erreicht werden, der den Kalkzusatz um das Hundertfache und mehr übertrifft. Man wird vielleicht annehmen dürfen, daß die Kalksalze durch chemische Bindung, die anderen durch „Aussalzen“" der Giftstoffe, d. h. durch Verschiebung der Lösungsverhältnisse entgiftend wirken. In derselben Weise wie die Kalksalze wirken Soda, Barium- und Zinksalze. Die giftaufhebende Wirkung der Kalksalze konnte noch in anderer Weise dargetan werden. Brachte man nämlich Bierhefe mit Weizenmehl in kalkreicher Bierwürze zusammen, so konnte kein Absterben der Hefezellen beobachtet werden. Wurde die Würze mit kalkreichem Leitungswasser verdünnt, so ergab sich dasselbe Gärbild. Verwandte man aber zum Verdünnen destilliertes Wasser, so trat sofort die intensive Giftwirkung des Weizenmehls zurage.

Über die Art und Wirkung des Giftstoffs können wir, abgesehen von der Gewißheit, daß er unter den Eiweißstoffen schlechthin zu suchen ist, bisher nur Vermutungen aussprechen, die bei den Mitarbeitern in verschiedener Richtung gehen. Ich persönlich neige mich der Ansicht zu, daß der Giftstoff unmittelbar giftig auf die Hefe wirkt. Henneb erg kam auf Grund einer Reihe von Untersuchungen zu einer anderen U̇berzeugung. Er fand, daß die Giftwirkung von Säuren, welche auf Bierhefe eine stärkere ist als auf Preßhefe, aufgehoben wird durch die Kalksalze. Er konnte weiter zeigen, daß Ammoniumsalze unter gewissen Umständen die Bierhefe abtöten, wenn auch in sehr viel längerer Zeit als Weizenmehl, daß ferner die Hefen in kalkfreien Nährlösungen verhältnismäßig schnell absterben, daß dagegen auch in den beiden letzten Fällen die Empfíndlichkeit der Hefen durch Zusatz von Kalksalzen aufgehoben wird. Aus alledem ist $H$ e $\mathbf{n} \mathbf{n} \theta \mathrm{b}$ e $\mathbf{r} \mathbf{g}$ den $\operatorname{Schluß}$ zu ziehen geneigt, daß bei allen den genannten Vergiftungserscheinungen der Hefe dieselben Gründe vorliegen, die in einer Säurebildung der Hefe zu suchen sind, durch welche die Hefe sich selbst vergiftet. Danach würde also auch die Vergiftung der Hefe durch unsere neuen Giftstoffe auf eine Säurebildung der Hefe durch
Umsetzung oder Aufspaltung gewisser Eiweißstoffe zurückzuführen, die Vergiftung also eine sekundäre sein. Welche von beiden Ansichten das Richtige trifft, müssen weitere Untersuchungen lehren. Es sei hier an die bekannte Oxalsäurebildung durch Hefe erinnert, die sich durch das Auftreten dér charakteristischen Krystalle des oxalsauren Kalkes in kalkhaltigen Gärlösungen zu erkennen gibt. Wenn man auch bisher die näheren Umstände dieser Frscheinung nicht erforschen konnte, so läßt sich doch mit ziemlicher Gewißheit sagen, daß der Grad der Oxalsáurebildung einmal abhängig ist von der Hefenrasse und weiter von den der Hefe dargebotenen Lebensbedingungen, vorzüglich den Nährstoffen. Die Menge der von der Hefe erzeugten Oxalsäure ist mitunter außerordentlich groß; so beobachtete $\mathrm{L}$ i n $\mathrm{d} n$ er eine Biertrübung, die nur von oxalsauren Kalkkrystallen herrührte. Durch übersehüssigen Kalk wird die Oxalsäure gebunden und unschädlich gemacht. Fehlt der Kalk und ist die Hefe gleichzeitig durch gewisse Lebensbedingungen gezwungen reichlich Oxalsäure hervorzubringen, so geht sie daran $\mathbf{z u}$ grunde. Es ließe sich denken, daß die von uns gefundenen Eiweißhefengifte solche Stoffe sind, welche die Hefe zu Oxalsäure verarbeiten oder aufspalten muB, und die letztere bei Abwesenheit von Kalk in Aktion tritt.

Ich habe bisher immer nur von der Bierhefe als Opfer der Wirkung der neuen Giftstoffe gesprochen und zwar deshalb, weil sie unser Hauptversuchsobjekt war; aber sie hat auch Leidensgefährten, denn ich konnte später zeigen, daß unter gewissen Umständen auch obergärige Brennerei- und Preßhefen der Giftwirkung erliegen, während $\mathrm{H}$ e n n e b e r g dasselbe für eine große Anzahl von verschiedenartigen Kultur- und wilden Heferassen nachwies. Immerhin blieb in allen Fällen die Bierhefe am empfindlichsten und ließ sich ihr diese Rasseneigentiumlichkeit bisher auch nicht durch besondere Züchtungsmaßnahmen abgewöhnen.

Eine Wirkung unserer Giftstoffe auf andere Mikroorganismen als Hefen haben wir bisher nicht ermitteln können.

M. H.! Sie sehen aus dem bisher Mitgeteilten, da $B$ wir das neue Hefegift genau genug kannten, um mit Aussicht auf Erfolg in den Maischen und Würzen der Gürungsgewerbe nach ihm zu fahnden. Wir kannten sein Verhalten gegen die chemischen und physiologischen Einflüsse des Mälzens, Darrens und Maischens und den Einfluß der Kalksalze des Maischwassers auf seine Wirkung. Wir haben diese Bahn auf verschiedenen Wegen betreten, die zum Teil bereits zu Erfolgen führten. Unsere Untersuchungen nach der praktischen Seite hin sind so zahlreich und die dabei auftretenden noch ungelösten Fragen so mannigfaltig, da $B$ ich heute nicht darauf einzugehen vermag, sondern mich mit einer kurzen Skizzierung unserer Arbeitsrichtungen und der bisherigen Erfolge begnügen muß.

Untersuchungen in Roggenmaische der Brennereien hat $\mathrm{E} 11 \mathrm{r}$ o d t angestellt und dabei gefunden, da $B$ hierin die Bierhefe unter gewissen Bedingungen der Getreidegiftwirkung erliegt, so da $B$ nunmehr ein weiterer Grund dafür gefunden ist, daß die Bierhefe in die Kornbrennereien keinen Eingang gefunden hat. Für obergärige Brennereihefen hat er 
dagegen bisher keine Giftempfindlichkeit in Roggenmaischen feststellen können.

H. La n ge und Ellrodt studierten die Möglichkeit einer Vergiftung der Hefe in der Bäckerei. Sie konnten eine solche nicht mit Gewißheit feststellen, kamen aber zu dem interessanten Ergebnis, daß sowohl bei Verwendung von Bäckereihefe wie von Bierhefe eine Verbesserung des Gebäckes erzielt wurde, wenn man dem aus destilliertem Wasser und Weizenmehl hergestellten Teig Kalksalze zusetzte. Es ist danach nicht unwahrscheinlich, daß die Hefe im Teig bei Mangel an Kalksalzen durch unsere Giftstoffe im Weizen an der Ausübung ihrer vollen Kräfte verhindert wird. Von mir im Verein mit $\mathrm{Sch} \ddot{\mathrm{u}} \mathrm{ck}$ ing sind die Verhältnisse der untergärigen Brauerei studiert worden. Auch hier ist es uns bisher nicht gelungen, das Hefengift in den Würzen nachzuweisen, aber wir haben wiederum neue interessante Wirkungen der Kalksalze im Brauwasser aufgedeckt, indem wir nachwiesen, daß der allgemein als indifferent oder gar schädlich geitende kohlensaure Kalk im Brauwasser unter Umständen einen äußerst günstigen Einfluß auf Hefe und Gärung in der Würze ausübt. Es gelang uns in Brauereien, die mit ungewöhnlich langer Gärzeit, schlechter Bruchbildung, mangelhaftem Absetzen der Hefe, lockerem Liegen der Hefensätze zu kämpfen hatten, diese Mängel durch Mitvermaischen von kohlensaurem Kalk zu beheben. Ob und wie weit hier die Wirkung des kohlensauren Kalkes auf einer Bindung der aus dem Malz stammenden Hefengifte beruht, werden wir hoffentlich demnächst mitteilen können.

Den Sehluß meiner heutigen Ausführungen soll die Mitteilung unserer neuesten Ergebnisse auf dem Gebiete der Hefengiftfrage bilden. Es ist mir nämlich gelungen, aus getrockneter Bier- und Preßhefe wässerige Auszüge zu gewinnen, die bei Gegenwart von Rohrzucker eine intensive Giftwirkung auf Bierhefe ausüben. Es scheint danach, daß die Hefe nicht nur gefährdet ist durch die Eiweißgifte in den Rohstoffen, sondern auch durch Stoffe gleicher Art, die ihrem eigenen Organismus angehören oder sich aus ihm unter gewissen Umständen bilden.

Es wird jetzt unsere Aufgabe sein, den Nachweis zu führen, daß eine Selbstvergiftung der Hefe durch ihre eigenen Eiwei@stoffe eintreten kann, und daß auf ihre Wirkung und auf diejenige der gleichgearteten Körper in den Rohstoffen der Gärungsgewerbe zahlreiche Mängel der Gärung zurückzuführen sind, die wir u. a. als stillstehende Gärung und Blasengärung in der Brauerei, als geringe Hefenernte in der Preßhefenfabrik, als niedrige, nicht durch Bakterienwirkung begründete Vergärung und endlich als Schaumgärung in der Brennerei beobachten. Wenn uns dieser Nachweis gelingt, so sind wir auf dem Wege zum Verständnis des Hefelebens im Gärbottich ein gut Stück weiter gekommen und können hoffen, daB wir dann auch Mittel und Wege finden werden, um allen den genannten Mängeln der Gärung, soweit sie auf unsere neuen Hefegifte zurückzuführen sind, nachdrücklich abzuhelfen.

\section{Über die Erscheinung der "Adsorption“, des „Alterns" und der „Gewöhnung“ bei Kolloiden.}

\author{
Von EDTARD Jordis.
}

(Eingeg. d. 11./7. 1908.)

Läßt man eine doppelte Umsetzung nach dem Schema $A B+C D=A D+B C$ vor sich gehen, so enthält der gebildete Körper AD nicht nur die Komponenten $\mathrm{A}$ und $\mathrm{D}$, die ihm nach der Formel zukommen, sondern auch noch Anteile von B und C; ist $\mathrm{AD}$ ein Kolloid, so tritt diese Erscheinung regelmäßig ein, doch findet sie sich ebenso bei Krystalloiden. Sie verursacht die großen Schwierigkeiten der Atomgewichtsbestimmungen, weil es nur unter Beobachtung besonderer Versuchsbedingungen gelingt, wirklich der Formel entsprechende reine Präparate darzustellen. Der Vorgang setzt schon bei der Bildung von Salzen einwertiger Basen und Säuren, wenn auch nur schwach, ein; mit der Wertigkeit der Komponenten nimmt er beträchtlich zu und erreicht bei den Kolloiden erhebliche Beträge. Dem Analytiker ist er wohl bekannt und bildet für ihn eine Quelle vieler Schwierigkeiten. Man bezeichnet ihn als ,Adsorption" und nimmt an, da 3 er rein mechanischer Art sei. Die ,Verunreinigung“" werde irgend wie von dem entstehenden Stoff ,eingeschlossen, mitgerissen " bzw. durch eine besondere in der Oberfläche der Körper wirkende Kraft festgehalten, ,adsorbiert“. Die Mitwirkung chemischer Verwandtschaft wird ansdrücklich bestritten, namentlich mit der Begründung, daß schon die geringe Menge der „Verunreinigung" und der Mangel stöchiometrischer Beziehungen die Möglichkeit chemischer Vorgänge ausschließe.

Die Adsorption wird bei Kolloiden, namentlich bei der Gelbildung, immer beobachtet und erreicht hier so hohe Beträge, daß man von nebensächlichen "Verunreinigungen" kaum mehr sprechen kann. Auch gelingt es bei diesen nicht mehr, wie bei Krystalloiden doch wenigstens in vielen Fällen, die "Verunreinigungen" durch Reinigungsverfahren, z. B. Auswaschen, fortzuschaffen. Dennoch wird auch hier der chemische Charakter der Adsorption durchaus geleugnet; auf der Annahme, daß es sich um rein physikalisch-mechanische Vorgänge handle, sind ja weitgehende und scharfsinnige Theorien aufgebaut worden. Der Umstand, daß die Adsorption bei Kolloiden in stärkerem Grade auftritt, als bei den Krystalloiden, gestattet, die Frage nach der Natur der Erscheinungen durch das Experiment aufzuklären. Arbeiten dieser Art sind seit einigen Jahren von verschiedenen Forschern ausgeführt worden und lassen gar keinen Zweifel mehr, daß es sich auch bei der Adsorption um normale chemische Reaktionen handelt. Die gegenteilige Auffassung ist irrig.

$\mathrm{Zu}$ derartigen Untersuchungen eignen sich vorzugsweise die Umsetzungen zwischen Salzen mehrwertiger anorganischer Basen und Säuren. Bei ihnen sind die adsorbierten Mengen besonders groß. Auch läßt sich die notwendige fortdauernde analytische Kontrolle der Vorgänge bequem durchführen. Hierzu ist es natürlich wichtig, Stoffe zu wählen, die leicht und scharf analytisch bestimmt 